Research Paper

\title{
Protracted Upregulation of Leptin and IGF1 is Associated with Activation of PI3K/Akt and JAK2 Pathway in Mouse Intestine after lonizing Radiation Exposure
}

\author{
Shubhankar Suman ${ }^{1}$, Bhaskar V. S. Kallakury², Albert J. Fornace Jr. ${ }^{1,3}$, Kamal Datta ${ }^{\circledR}$ \\ 1. Department of Biochemistry and Molecular \& Cellular Biology and Lombardi Comprehensive Cancer Center, Georgetown University Medical \\ Center, Washington DC, USA. \\ 2. Department of Pathology, Georgetown University Medical Center, Washington DC, USA. \\ 3. Center of Excellence in Genomic Medicine Research (CEGMR), King Abdulaziz University, Jeddah, Saudi Arabia.
}

$\square$ Corresponding author: Kamal Datta, M.D., Associate Professor, Department of Biochemistry and Molecular \& Cellular Biology, Georgetown University, Research Building, Room E518, 3970 Reservoir Rd., NW, Washington, DC 20057, USA. Phone: 202-687-7956, Fax: 202-687-3140, Email: kd257@georgetown.edu

() 2015 Ivyspring International Publisher. Reproduction is permitted for personal, noncommercial use, provided that the article is in whole, unmodified, and properly cited. See http:/ /ivyspring.com/terms for terms and conditions.

Received: 2014.09.30; Accepted: 2014.12.25; Published: 2015.01.20

\begin{abstract}
lonizing radiation is a known risk factor for gastrointestinal $(\mathrm{Gl})$ pathologies including cancer. Hormones and related signaling crosstalk, which could contribute to radiation-induced persistent pathophysiologic changes in the small intestine and colon, remain to be explored. The current study assessed perturbation of $\mathrm{Gl}$ homeostasis-related hormones and signaling pathways at the systemic as well as at the tissue level in small intestine and colon. Mice (6-8 week old C57BL/6]) were exposed to 2 Gy $\mathrm{Y}$ radiation, serum and tissue samples were collected, and insulin like growth factor 1 (IGF-1) and leptin signaling were assessed two or twelve months after radiation exposure. Serum levels of IGF-1, IGF binding protein 3 (IGFBP3), leptin, and adiponectin were altered at these times after irradiation. Radiation was associated with increased IGFl receptor (IGFIR) and obesity (leptin) receptor (Ob-R), decreased adiponectin receptor 1 (Adipo-RI) and 2 (Adipo-R2), and increased $\mathrm{Ki}-67$ levels in small intestine and colon at both time points. Immunoblot analysis further showed increased IGFIR and Ob-R, and decreased Adipo-R2. Additionally, upregulation of PI3K/Akt and JAK2 signaling, which are downstream of IGF1 and leptin, was also observed in irradiated samples at both time points. These results when considered along with increased cell proliferation in the small intestine and colon demonstrate for the first time that ionizing radiation can persistently increase IGFI and leptin and activate downstream proliferative pathways, which may contribute to $\mathrm{Gl}$ functional alterations and carcinogenesis.
\end{abstract}

Key words: ionizing radiation; colorectal cancer; leptin; insulin like growth factor 1 ; intestinal homeostasis

\section{Introduction}

Epidemiological studies in atomic bomb survivors and in nuclear facility workers have implicated radiation exposure as a risk factor for cancer as well as non-cancer gastrointestinal (GI) pathologies in human [1-5]. A large body of evidence supports the fact that hormones such as insulin like growth factor 1 (IGF1), leptin, and adiponectin regulate GI epithelial cell homeostasis and metabolism, and deregulation of these hormones have been reported to promote cancer and non-cancer diseases in the GI tract [6-13]. Indeed, a number of case control and prospective studies have reported altered IGF1, leptin, and adiponectin levels in serum as risk factors for colon cancer $[7,13,14]$. It is now established that IGF1 through its stimulatory effects on growth and proliferation and inhibitory effects on cell death plays critical roles in initiation 
and promotion of disease processes including colon cancer $[15,16]$. Cellular effects of IGF1 are mediated through its membrane-bound receptor IGF1R, which is expressed in normal GI epithelial cells as well as in colon cancer cells, to insulin receptor substrate (IRS1) and then to PI3K/Akt and its downstream effectors. Activities of IGF1 are further regulated by IGF binding protein 3 (IGFBP3), which binds to IGF1 and modulates levels of free bioactive IGF1 $[15,16]$. In addition to IGF1, two adipokines secreted from adipocytes, leptin and adiponectin, play important roles in maintaining GI homeostasis. Leptin, on one hand, is known to modulate absorption of nutrients in GI tract, and, on the other, it is also involved in promoting cellular growth and proliferation, inhibiting apoptosis, and stimulating pro-inflammatory responses in small and large intestine [17-19]. At the molecular level, leptin mediates its growth and proliferative effects via obesity receptor (Ob-R) to activate survival pathways such as JAK/STAT, PI3K/Akt, and MAPK $[20,21]$. In contrast, adiponectin through its two receptors (adiponectin receptor 1 or Adipo-R1 and adiponectin receptor 2 or Adipo-R2) exerts negative effects on proliferation and inflammation, and decreased expression of Adipo-R1 and Adipo-R2 is known to enhance proliferative signaling in GI tissues [10, 13, 22-25]. However, despite the fact that both radiation exposure and hormonal perturbations have separately been linked to intestinal diseases and colon cancer, we are yet to elucidate the complete spectrum of hormones and associated signaling pathway alterations involved in radiation-induced long-term pathophysiologic changes in small intestine and colon. We believe ours is the first report showing increased IGF1 and leptin levels in serum, and upregulated receptors and associated downstream signaling pathways in tissues long term ( 2 and 12 months after exposure) after a modest dose of $\gamma$ rays; these observations are suggestive of involvement of IGF1 and leptin signaling in radiation-associated long-term GI consequences.

\section{Materials and methods}

\section{Animal care and irradiation.}

Six- to eight-week-old female C57BL/6J mice (Jackson Laboratories, Bar Harbor, ME) were maintained in $12 \mathrm{~h}$ dark and light cycle at $22{ }^{\circ} \mathrm{C}$ in $50 \%$ humidity and provided certified rodent diet along with filtered water ad libitum. Mice ( $\mathrm{n}=10$ mice per study group) were exposed to 2 Gy whole-body $Y$ radiation using a ${ }^{137} \mathrm{C}$ s source. The radiation dose of 2 Gy was chosen because it is a moderately low sub-lethal dose and it is also a commonly used daily fraction in fractionated radiotherapy [26].
Post-irradiation, mice were returned to their home cages and monitored regularly and irradiation experiments were repeated three times and control mice were sham irradiated. All animal procedures were performed according to protocols approved by the Institutional Animal Care and Use Committees (IACUC) at Georgetown University. For our research, we followed Guide for the Care and Use of Laboratory Animals, prepared by the Institute of Laboratory Animal Resources, National Research Council, and U.S. National Academy of Sciences.

\section{Tissue and serum collection.}

Serum, and small intestine and colon tissues were collected two or twelve months after radiation for further analysis. Mice were euthanized as per approved protocol using $\mathrm{CO}_{2}$ asphyxiation and blood samples were collected by cardiac puncture immediately after euthanasia using sterile $1 \mathrm{ml}$ syringes and $25 \mathrm{G}$ needles. Serum was flash frozen in liquid $\mathrm{N}_{2}$, and kept at $-80^{\circ} \mathrm{C}$ for further use. Sections $(3 \mathrm{~cm})$ from the jejunum-ileum area and colon were surgically dissected out from each mouse, fixed in $10 \%$ buffered formalin, and $4 \mu \mathrm{m}$ thick sections were prepared after paraffin embedding for histology and immunohistochemistry.

\section{Serum hormone estimation.}

Serum IGF1 concentrations were measured using IGF-1 Quantikine ELISA kit (Cat\# MG100, R\&D Systems, Minneapolis, MN) according to the manufacturer's protocol. For each mouse sample, measurement was performed in duplicate and the assay has a detection sensitivity of $8.4 \mathrm{pg} / \mathrm{ml}$ with $<0.5 \%$ cross-reactivity with available related molecules.

Serum IGFBP3 concentrations were measured using IGFBP3 ELISA kit (Abcam, Ab100692, Cambridge, MA) according to the manufacturer's protocol. Measurements were performed in duplicates and the assay has a detection sensitivity of $<70 \mathrm{pg} / \mathrm{ml}$. Molar ratio of IGF1/IGEBP3 was calculated as per procedure described earlier [9]. Serum leptin concentrations were measured in duplicate using leptin ELISA kit (Cat\#KMC2281, Life Technologies, Grand Island, NY) according to the manufacturer's protocol. The assay has a detection sensitivity of $<50 \mathrm{pg} / \mathrm{ml}$. Serum adiponectin concentrations were measured in duplicate using adiponectin ELISA kit (Cat\# KMP0041, Life Technologies, Grand Island, NY) according to the manufacturer's protocol. This assay has a detection sensitivity of $<50 \mathrm{pg} / \mathrm{ml}$.

\section{Immunoblot analysis.}

Frozen small intestine and colon tissue samples from 5 mice were pooled, homogenized in ice-cold 
lysis buffer ( $0.5 \%$ sodium deoxycholate; $0.5 \%$ NP-40; $10 \mathrm{mM}$ EDTA in PBS) containing protease inhibitor cocktail (Sigma-Aldrich, St. Louis, MO), and centrifuged at $12000 \mathrm{xg}$ at $4{ }^{\circ} \mathrm{C}$ for $15 \mathrm{~min}$. Immunoblots were performed using appropriate primary antibodies (IGF1R: dilution-1:200, Sc7952, Santa Cruz Biotechnology, Dallas, TX; IRS1: dilution-1:500, 611394, BD Biosciences, San Jose, CA; p85: dilution-1:500, 4292S, Cell Signaling Technology, Danvers, MA; AKT: dilution-1:500, Sc5298, Santa Cruz Biotechnology; phospho-AKT: dilution-1:500, 9277S, Cell Signaling Technology; Ob-R: dilution-1:200, Sc1834, Santa Cruz Biotechnology; AdipoR2: dilution-1:200, Sc99184, Santa Cruz Biotechnology, JAK2: dilution-1:200, Sc7229, Santa Cruz Biotechnology; phospho-JAK2: dilution-1:200, Sc21870, Santa Cruz Biotechnology; $\beta$-Actin: dilution-1:2500, Sc47778, Santa Cruz Biotechnology). Immunoblot membranes were developed using horseradish peroxidase (HRP) conjugated secondary antibody and enhanced chemiluminescence (ECL) detection system (Thermo Fisher Scientific, Rockville, MD). Images were captured on x-ray films and scanned images were used for densitometric quantification by ImageJ v1.46 software (National Institutes of Health, Bethesda, MD). Band intensity was normalized to $\beta$-actin band intensity in respective column, results are expressed as fold change (irradiated/control) in the irradiated group relative to sham-irradiated controls, and representative images are shown in the results.

\section{Histology and immunohistochemistry.}

Small intestine and colon sections were stained with hematoxylin and eosin (H\&E) using standard protocol for histologic analysis. Morphometric analysis of the crypts and villi was also performed on these sections as described previously [27]. Briefly, images were captured using bright field microscopy at $4 \mathrm{X}$ microscopic magnification and heights of the five well oriented crypt-villus units in small intestine and crypts in colon were measured in each section $(n=10$ sections from ten mice per group and total 50 units were measured in each group) by an observer blinded to the treatment groups. The measurement was performed using ImageJ v1.46 software. Furthermore, a pathologist, also blinded to the treatment groups, performed histologic analysis of the stained sections for inflammatory cells and tumors. Immunostaining ( $n=5$ mice per group) for Ki-67 (dilution-1:50, Sc15402, Santa Cruz Biotechnology), IGF1R (dilution-1:50, sc-7952, Santa Cruz Biotechnology), Adipo-R1 (dilution 1:50, sc-99183, Santa Cruz Biotechnology), Adipo-R2 (dilution 1:50, sc-99184, Santa Cruz Biotechnology), and Ob-R (dilution-1:40, sc-1834, Santa Cruz Biotechnology) was performed following antigen re- trieval using citrate buffer ( $\mathrm{pH}$ 6.0). SuperPicture $3^{\text {rd }}$-generation IHC detection kit (Invitrogen, Carlsbad, CA) was used for signal detection and color development. Five slides from five mice in each group were stained for each protein and a representative image from one animal is presented in results. To determine the specificity of the staining, appropriate controls were run in parallel with the experimental slides.

\section{Immunohistochemistry quantification and statistical analysis.}

Images from anatomically similar regions of the small intestine and colon were captured by bright field microscopy at 20X microscopic magnification, and ten images from randomly selected visual fields were captured from each slide for quantification. While Ki-67 positive cells were counted using color deconvolution as well as Image-based Tool for Counting Nuclei (ITCN) plug-ins, staining intensity of IGF1R, Adipo-R1, Adipo-R2, and Ob-R were analyzed using color deconvolution feature of the ImageJ v1.46 software as per protocol described earlier [28-30]. To determine differences between the two groups, statistical analysis was performed with a two-tailed paired Student's $t$ test and $p<0.05$ was taken as statistically significant. Error bars represent mean \pm standard errors of the mean (SEM).

\section{Results}

\section{Radiation exposure increased serum IGF I levels, decreased IGFBP3, and increased IGFIR.}

Compared to sham irradiated controls, a small but significant increase in serum IGF1 was observed 2 months after irradiation (Figure 1A). Conversely, serum IGFBP3 was significantly decreased 2 months after irradiation (Figure 1B). Consequent to increased IGF1 and decreased IGFBP3, we observed a significantly increased IGF1/IGFBP3 molar ratio suggestive of increased free IGF1 (Figure 1C). Furthermore, serum IGF1 level was increased and the IGFBP3 level was decreased leading to increased IGF1/IGFBP3 molar ratio even 12 months after radiation exposure (Figure 1D, E, and F). Considering that greater serum hormonal alterations were observed at the 12-month time point relative to 2-month, immunohistochemical and immunoblot analysis were performed in the 12-month samples. IGF1R expression was increased in irradiated samples relative to controls in small intestine (Figure 1G and $\mathrm{H}$ ) and in colon (Figure 1I and J) at the 12-month time point. 


\section{Serum leptin levels were increased after irra- diation.}

Serum adiponectin levels were not significantly altered 2 months after radiation exposure relative to controls (Figure 2A). However, serum leptin levels were significantly increased at this time, relative to sham-irradiated controls (Figure 2B). Since, the leptin/adiponectin ratio (L/A ratio) is considered as an additional risk factor for CRC, we calculated the L/A ratio, and found significantly higher ratio at 2-month post-irradiation relative to controls (Figure 2C). Also, serum adiponectin levels remained unchanged (Figure 2D), but leptin levels and L/A ratio increased significantly (Figure 2E and $\mathbf{F}$ ) in the 12-month post-irradiation samples.
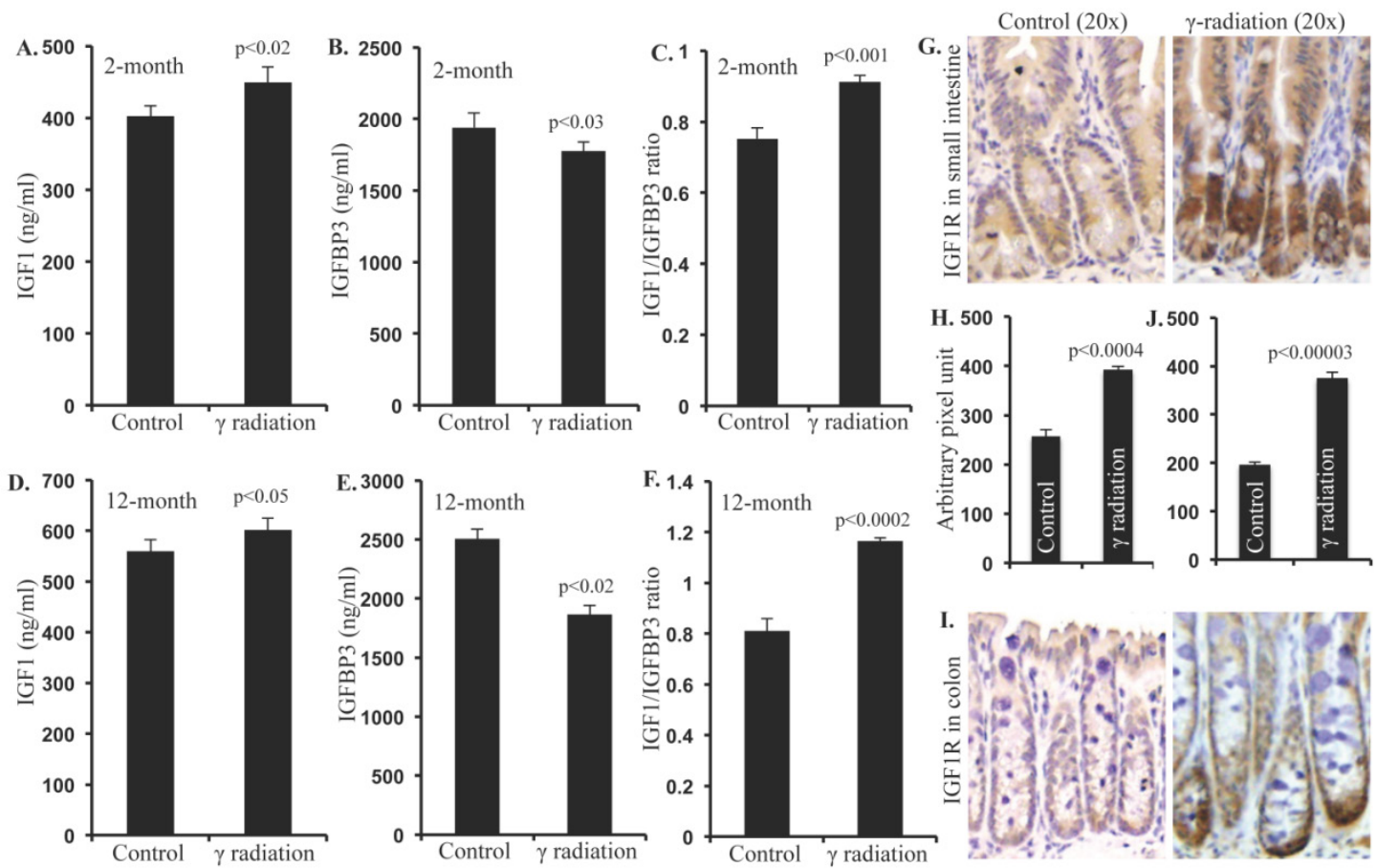

Figure 1. Radiation-induced long-term changes in IGFI, IGFBP3, and IGF1R. A) Serum IGF1 levels at 2 months post-exposure, B) Serum IGFBP3 levels at 2 months post-exposure, C) Molar ratio of IGF1/IGFBP3 representing free IGF1 levels at 2 months post-exposure, D) Serum IGF1 levels at 12 months post-exposure, E) Serum IGFBP3 levels at 12 months post-exposure, F) Molar ratio of IFG1/IGFBP3 at 12 months post-exposure. G) Representative images of IGF1R immunostained small intestine sections at 12 months post-irradiation. H) Quantitation of IGFIR signal intensity in small intestine sections. I) Representative images of IGFIR immunostained colon sections at 12 months post-irradiation. J) Quantitation of IGFIR signal intensity in colon sections. Error bars represent mean \pm SEM and $p<0.05$ was considered significant, compared to sham-irradiated control.

Figure 2. Serum levels of adiponectin and leptin were altered 2 and 12 months after radiation exposure. A) Serum adiponectin levels at 2 months post-exposure, B) Serum leptin levels at 2 months post-exposure, C) Molar ratio of leptin/adiponectin (L/A) at 2 months post-exposure, D) Serum adiponectin levels at 12 months post-exposure, E) Serum leptin levels at 12 months post-exposure, F) Molar ratio of leptin/adiponectin (L/A) at 12 months post-exposure. Note that $y$-axis scale differs panels $B$ versus $E$ and $C$ versus $F$. Error bars represent mean \pm SEM and $\mathrm{p}<0.05$ was considered significant, compared to sham irradiated control.
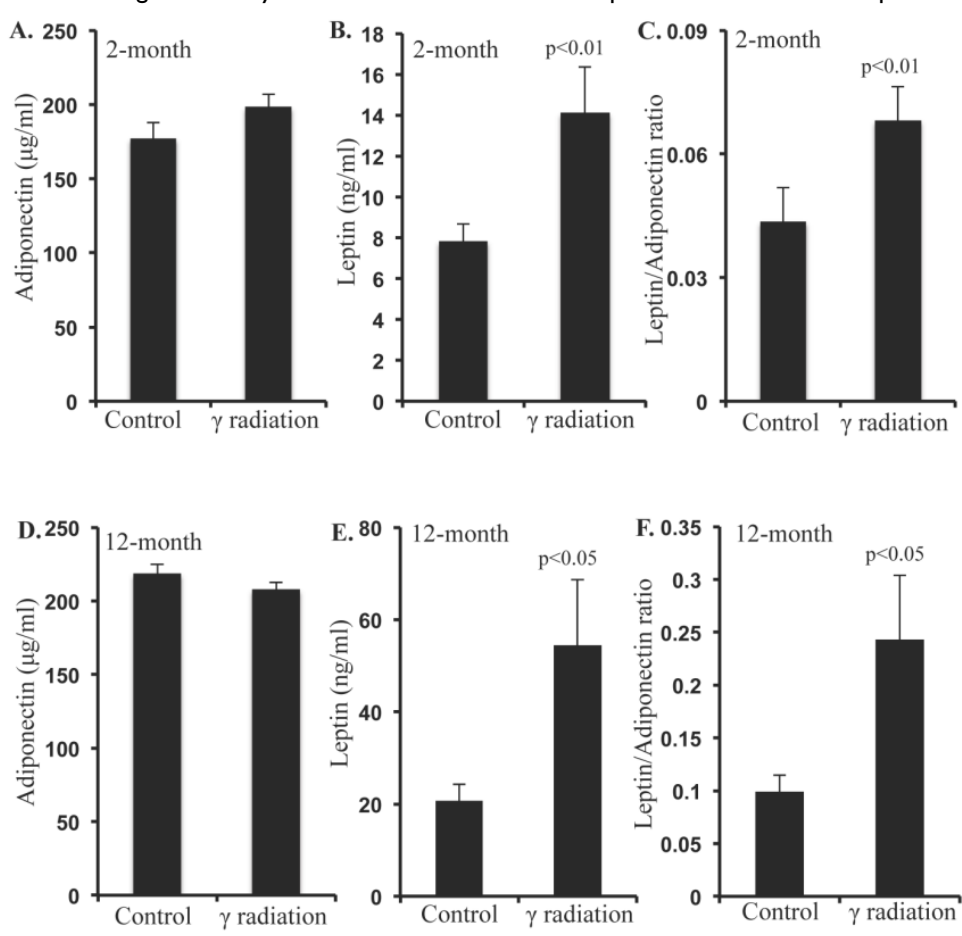

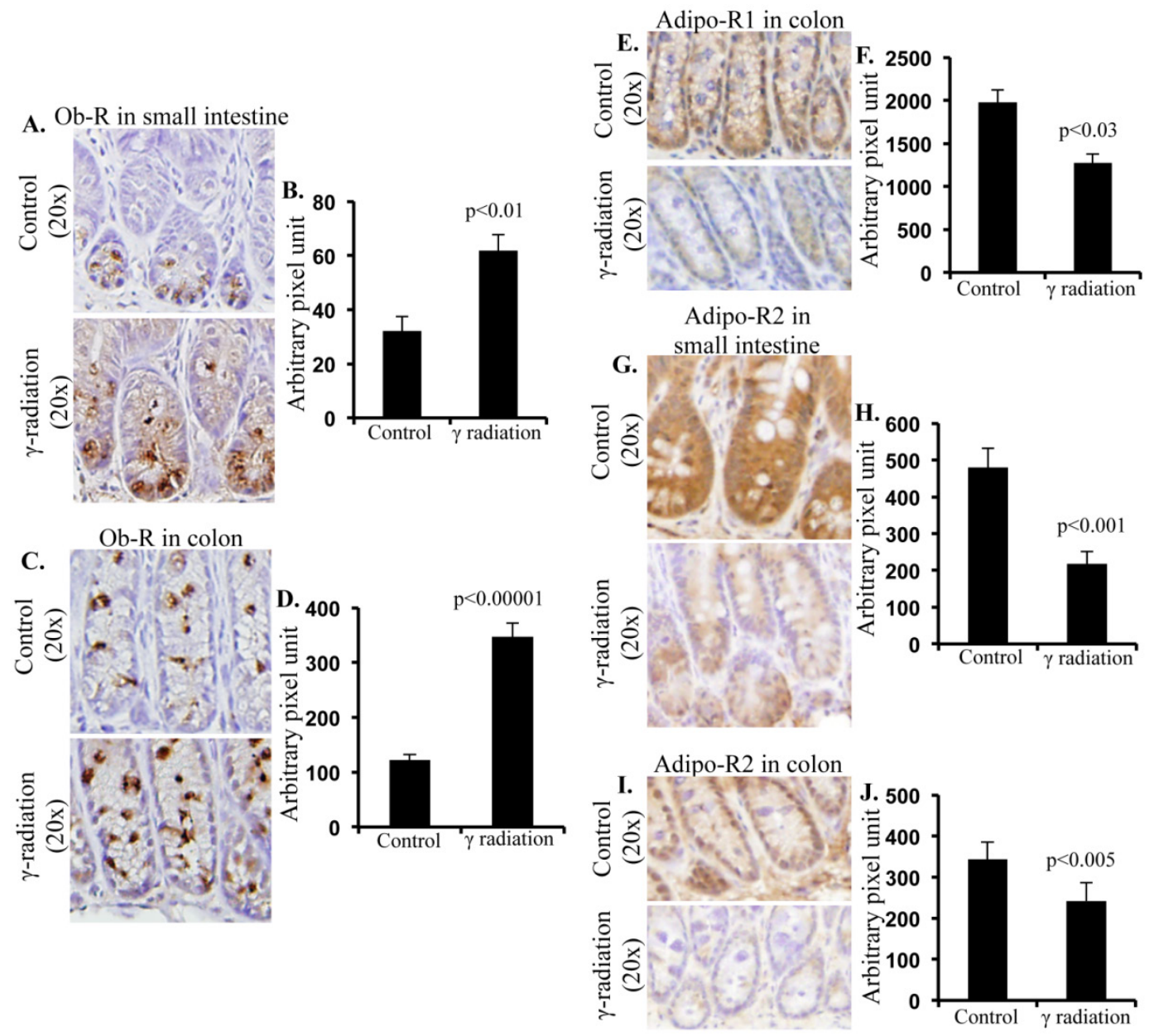

Figure 3. Radiation increased Ob-R, and decreased Adipo-R1 and Adipo-R2 levels in small intestine and colon 12 months after $\gamma$ irradiation. A) Representative images of Ob-R immunostained small intestinal sections. B) Quantitation of Ob-R signal intensity in small intestinal sections. C) Representative images of Ob-R-immunostained colon sections. D) Quantitation of Ob-R signal intensity in colon sections. E) Representative images of Adipo-R1-immunostained colon section. F) Quantitation of Adipo-R1 signal intensity in colon sections. G) Representative images of Adipo-R2-immunostained small intestine sections. H) Quantitation of Adipo-R2 signal intensity in small intestine sections. I) Representative images of Adipo-R2 immunostained colon sections. J) Quantitation of Adipo-R2 signal intensity in colon sections. Error bars represent mean \pm SEM and $\mathrm{p}<0.05$ was considered significant, compared to sham irradiated control.

Irradiation increased Ob-R but decreased Adipo-R1 and -R2 levels in small intestine and colon 12 months after irradiation.

Increased $\mathrm{Ob}-\mathrm{R}$ immunostaining was observed in small intestine sections (Figure 3A) and quantification of immunostaining showed significantly higher $\mathrm{Ob}-\mathrm{R}$ in irradiated samples relative to sham-irradiated controls (Figure 3B). Increased Ob-R expression was also observed in colon sections of irradiated mice (Figure 3C) and levels of Ob-R were significantly higher in irradiated vs. sham-irradiated mice (Figure 3D). In addition, Adipo-R1 immunostaining was decreased in colon samples of irradiated mice (Figure 3E). Quantification showed statistically significant downregulation of Adipo-R1 in colon (Figure 3F) relative to controls. However, we did not observe any alterations in Adipo-R1 level in small intestine sections of irradiated mice (data not shown). Also, Adipo-R2 in small intestine (Figure 3G) and colon (Figure
3I) was decreased after irradiation. Quantification showed significant downregulation of Adipo-R2 in small intestine (Figure 3H) and colon (Figure 3J) after irradiation.

\section{Radiation-induced IGFI and leptin activated downstream proliferative signaling pathways in small intestine and colon 12 months after irradiation.}

Immunoblot analysis of small intestinal tissue showed significantly increased IGF1R, insulin receptor substrate 1 (IRS1), p85, Akt, and phospho-Akt (p-Akt) levels after radiation exposure (Figure 4A and B). Importantly, we also observed significantly increased Ob-R, decreased Adipo-R2, and increased JAK2 and phospho-JAK2 (p-JAK2) in small intestinal tissues of irradiated mice relative to sham-irradiated controls (Figure 4A and B). Immunoblot analysis of colon tissue also showed significantly increased IGF1R, IRS1, p85, Akt, and p-Akt levels after radiation 
exposure (Figure 5A and B). Notably, significantly increased Ob-R, decreased Adipo-R2, and increased JAK2 and p-JAK2 was also observed in colon tissues of irradiated mice relative to sham-irradiated controls (Figure 5A and B). Immunohistochemical analysis of Ki-67 positive cells in small intestine (Figure 5C and D) and colon (Figure 5E and F) tissues of irradiated mice showed significantly higher expression than in
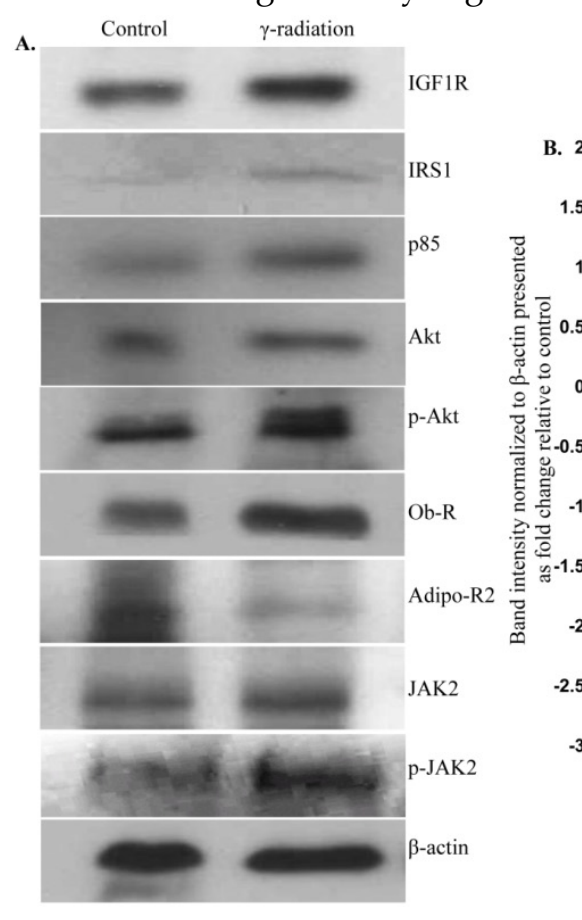

the control samples. Histologic analysis of small intestine and colon sections at this time point did not show significant difference in inflammatory cell numbers in irradiated groups relative to controls (data not shown). Morphometric analysis showed increased height of the crypt-villus units in small intestine (Figure 6A and B) and crypts in colon (Figure 6C and D).

Figure 5. Immunoblot analysis of IGFI and leptin signaling in colon 12 months after radiation exposure. A) Representative images of IGFIR, IRSI, p85, Akt, p-Akt, Ob-R, Adipo-R2, JAK2, p-JAK2, and $\beta$-actin immunoblots in colon samples. B) Quantification of immunoblot band intensities normalized to $\beta$-actin band intensity presented as fold change (irradiated/control) in irradiated groups relative to controls. C) Representative images of $\mathrm{Ki}-67$ immunostained small intestine sections. D) Quantitation of $\mathrm{Ki}-67$ positive nuclei in small intestine. E) Representative images of Ki-67-immunostained colon sections. F) Quantitation of Ki-67 positive nuclei in colon. Error bars represent mean \pm SEM and $p<0.05$ was considered significant, compared to sham irradiated control.
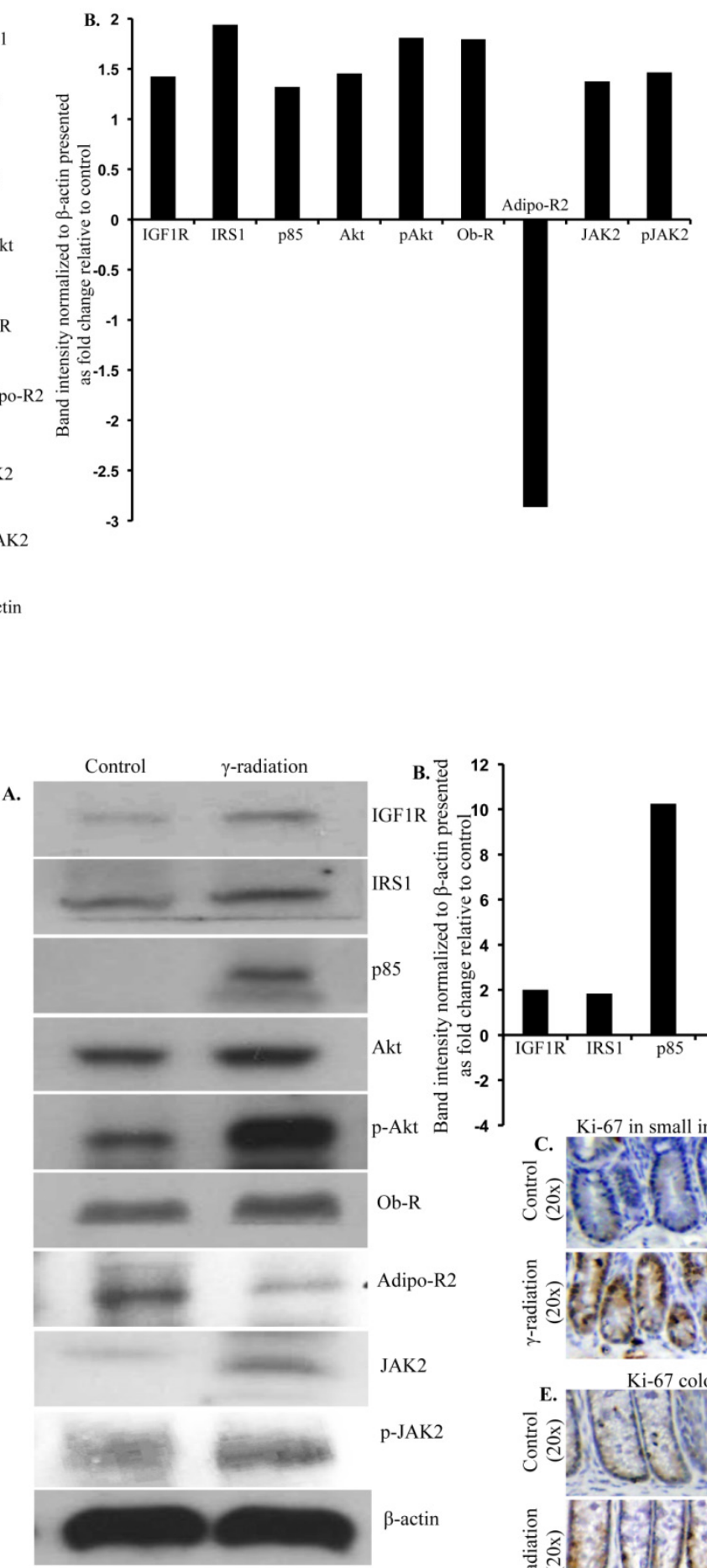

Figure 4. Immunoblot analysis of IGFI and leptin signaling in small intestine 12 months after radiation exposure. A) Representative images of IGFIR, IRSI, p85, Akt, p-Akt, Ob-R, Adipo-R2, JAK2, $\mathrm{p}-\mathrm{AK} 2$, and $\beta$-actin immunoblots in small intestinal samples. B) Quantification of small intestinal immunoblot band intensities normalized to $\beta$-actin band intensity presented as fold change (irradiated/control) in irradiated groups relative to controls. 

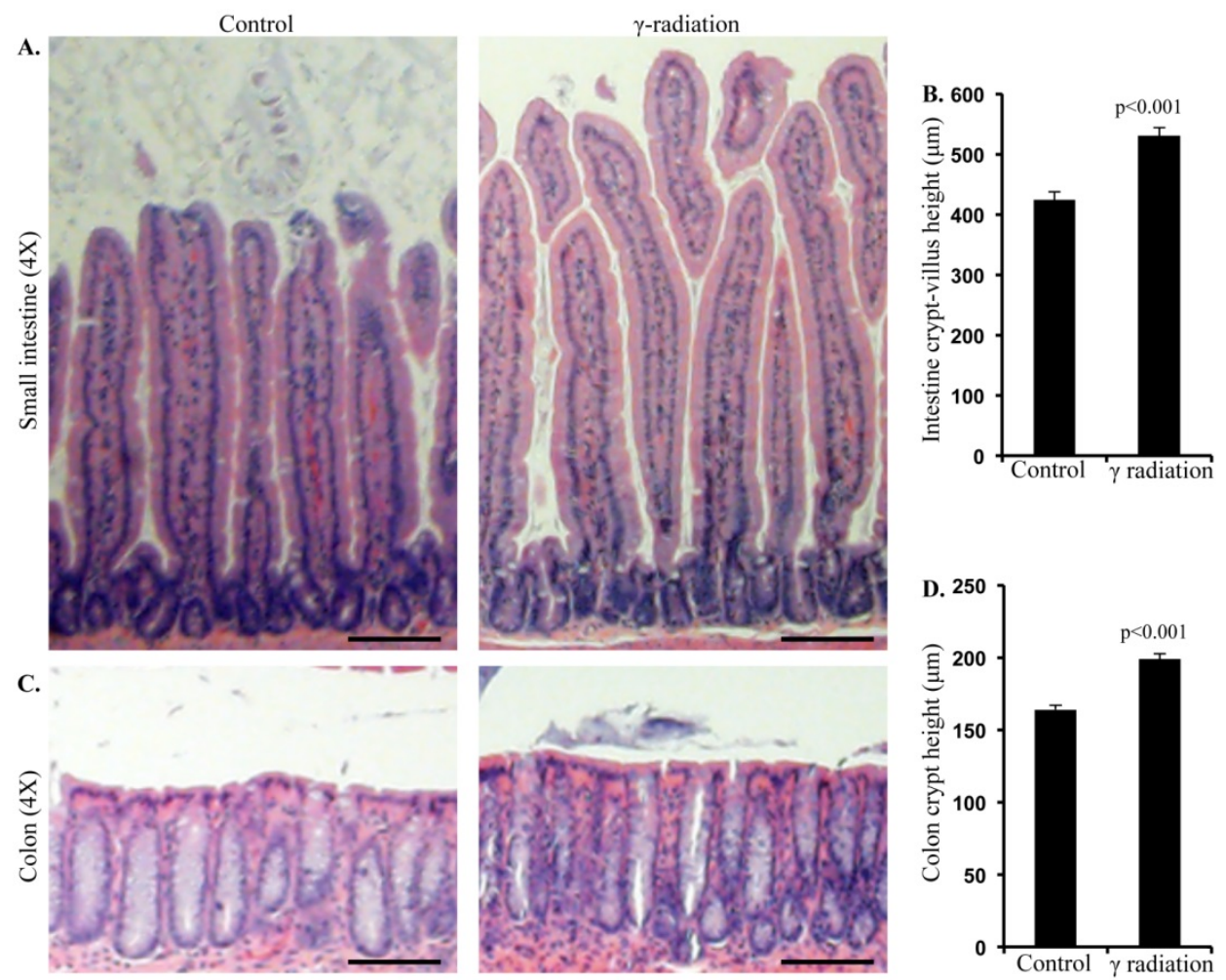

Figure 6. Radiation exposure was associated with altered mucosal morphology. A) Representative images showing small intestinal crypt-villus height in control and irradiated mice. B) Quantification of small intestinal crypt-villus height. C) Representative images showing colon crypt height in control and irradiated mice. D) Quantification of colon crypt height. Error bars represent mean \pm SEM and $\mathrm{p}<0.05$ was considered significant, compared to sham irradiated control and scale bars $=$ $100 \mu \mathrm{m}$.

\section{Discussion}

While radiation exposure is known to be a risk factor for chronic intestinal diseases such as malabsorption, intestinal inflammatory conditions, and colon cancer [1, 2, 7-11], our knowledge with respect to tissue specific molecular pathway alterations especially hormonal pathways that are associated with long-term sequela of radiation exposures in the GI tract are limited. Here we demonstrated that exposure to a $\gamma$ radiation dose (2 Gy) commonly used as a daily fraction in radiotherapy resulted in long-term alterations of hormones, IGF1, leptin, and adiponectin, which are involved in the homeostasis of the GI tract. An interesting point to note in the current study is that in control mice there was an age related increasing trend in serum levels of IGF1, leptin, and adiponectin with increased levels in 12-month relative to 2-month control mice. While age-related increased IGF1 and adiponectin levels were not statistically significant, leptin levels were significantly higher in 12-month relative to 2-month control samples and are in agreement with earlier reports [31, 32]. We also showed that radiation exposure persistently increased IGF1R and Ob-R, and decreased Adipo-R1 and Adipo-R2 expression in mouse small intestine and colon. We believe altered expression of receptors is working in tandem (summarized in Figure 7) with perturbed hormonal levels towards activation of downstream proliferative signaling pathways leading to increased intestinal and colonic epithelial cell proliferation indicated by increased Ki-67 staining and mucosal growth. In the GI tract, epithelial cell proliferation, differentiation, and migration are tightly regulated and crypts with stem and progenitor cells are critical for the regular cellular turnover in small intestine as well as in colon [33]. In this regard, a closer examination of our immunoprobed tissue sections apart from showing altered expressions of IGF1R, Ob-R, Adipo-R1 and R2, and Ki-67 also show that these altered expression levels are predominantly localized in the crypts suggesting that stem and progenitor cells are affected. However, additional studies using stem cell specific mouse models [34] would aid in delineating roles of stem cells in IGF1 and leptin signaling-associated changes and their potential for initiating pathophysiological processes in small intestine and colon.

There has been considerable research linking IGF1 to regulation of GI cell proliferation, and differentiation and thus to maintenance of GI homeostasis and optimal functioning. Furthermore, IGF1 due to its mitogenic and anti-apoptotic properties is also linked to colon cancer initiation and promotion and in- 
creased IGF1 and IGF1/IGFBP3 molar ratio is reported to be a risk factor for colon cancer [14, 16]. However, the roles of IGF1 signaling in radiation-induced GI disorders have not yet been explored. Considering that the bioavailability of IGF1 is in part regulated by IGFBP3, increased IGF1 and IGF1/IGFBP3 molar ratio observed in our study even 12 months after irradiation is indicative of increased free IGF1 available to bind to its receptor, IGF1R. Small intestine and colon epithelial cells express IGF1R and upon IGF1 binding, the receptor-ligand internalizes to activate its downstream effector pathways to promote cell proliferation [35]. While IGF1-binding-mediated activation of IGF1R, a member of the type II receptor tyrosine kinase family, is known to upregulate IGF1R adaptor protein, IRS1 [35], the current study is indicative of a connection between radiation exposures and the IGF1/IGF1R/IRS1 axis and has implications for radiation-related colorectal carcinogenesis. We also demonstrated that radiation exposure led to activation of pro-proliferative PI3K/Akt pathway, which is considered to be downstream of IRS1 [35]. Although, IGF1/IGF1R/IRS1-mediated PI3K/Akt signaling axis has been reported earlier [35], our study is suggestive of a relationship between radiation and PI3K/Akt via IGF1/IRS1, which could be contributing to observed cell proliferation. Importantly, a persistently decreasing trend of IGFBP3 with increasing trend of IGF1 level, we speculate, is providing further impetus to cell proliferation through greater bioavaiability of free IGF1 to bind to IGF1R. Interestingly, IGFBP3 itself has been shown to promote apoptosis via its direct effects on target cells independent of IGF1 [15] and chronic low IGFBP3 level observed in our study could reinforce proliferative effects of IGF1.

Increased levels of leptin, similar to IGF1, have also been reported to have a positive effect on cellular proliferation, and a negative effect on apoptosis, a corner stone of functional alterations as well as carcinogenesis [19]. Importantly, in the current study, increased leptin level was associated with increased expression of its receptor (Ob-R) as well as activation of downstream JAK2 pathway in small intestine as well as in colon providing further credence to observed cell proliferation. On the other hand, crosstalk between leptin and IGF1 pathway via JAK2 and IRS1 has been reported earlier [36, 37]. Consequently, when considered along with the fact that leptin has also been reported to promote IGF1 expression via JAK2/STAT5 pathway [38], our results of upregulated IGF1 and leptin support the notion that the radiation-induced persistent proliferative signal was in part due to co-operation between leptin and IGF1 pathways. Adiponectin is known to oppose prolifera- tive signals of IGF1 and leptin [39, 40] and our results of decreased adiponectin levels along with decreased Adio-R1 and Adipo-R2 expression could further contribute to cell proliferation through reduced antagonism to leptin and IGF1 signaling.

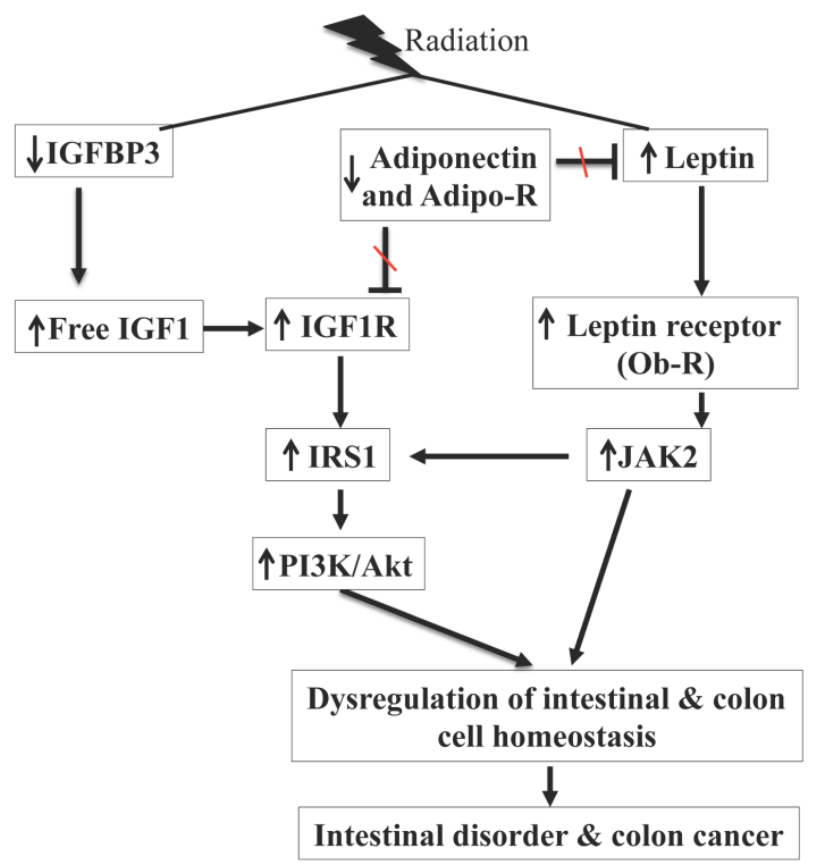

Figure 7. Summary of radiation-induced persistent alterations of IGFI and leptin signaling pathways observed in our study. Radiation exposure was associated with decreased IGFBP3, increased IGFI and IGFIR, and activation of PI3K/Akt pathway via IRSI. Radiation exposure also decreased adiponectin and Adipo-R, increased leptin and Ob-R, and activated JAK2 pathway. Adiponectin antagonizes IGFI and leptin, and decreased adiponectin and its receptors are expected to further promote IGFI and leptin signaling through reduced antagonism. There is also existence of crosstalk between JAK2 and PI3K/Akt via IRS1 and combined activation of these two pathways is contributing to intestinal cell proliferation with potential for intestinal disorder and colon cancer.

Both leptin and IGF1 are known to influence cellular energy metabolism and are considered markers of obesity [7, 12]. Conversely, radiation has also been reported to alter cellular metabolism especially at non-lethal doses [41, 42] and a number studies reported increased adiposity and body weight after radiation exposure [43-45]. Our study, in agreement with what has been reported in the literature, did note an increasing trend in the body weight of 12-month post-irradiated mice relative to sham-irradiated controls. However, increased body weight (irradiated vs. sham-irradiated) was not statistically significant (data not shown). Nonetheless, altered body weight trend attains importance in light of activated IGF1 and leptin signaling, which are known to link two important cellular processes, metabolism and proliferation $[14,16]$. Increased metabolism is essential to meet increased energy demand of the expanding cell population and radiation-induced 
activation of IGF1 and leptin signaling was associated with increased cell proliferation evidenced not only by increased Ki-67 staining but also by increased mucosal height shown in the morphometric analysis of the H\&E stained small intestine and colon sections of irradiated mice [27]. Increased cell number observed in the current study may not be categorized as compensatory proliferation, which occurs due to signaling from high dose radiation-induced apoptotic cells or from apoptotic but death-blocked cells [46]. Considering that TUNEL staining did not show increased cell death in irradiated mice exposed to a sub-lethal 2 Gy dose relative to controls exposed to sham-radiation (data not shown and [28]), increased proliferation leading to epithelial cell hyperplasia, we believe, is due to activation of IGF1/leptin signaling and is in line with what has been reported earlier about the effects of these two hormones on GI cell proliferation [47-49]. Furthermore, pro-proliferative effects of IGF1 and leptin pathways have also been implicated in intestinal disorder and colon cancer [50, 51]. Notably, we did not observe any intestinal and colon tumorigenesis in these post-irradiated wild type mice within the duration of our study (data not shown). Also, histologic examination and immunoprobing for inflammatory cells did not show any difference between irradiated and controls suggesting that the radiation dose used in the current study did not induce inflammatory cell infiltration mimicking inflammatory bowel disease (data not shown). However, we believe that additional studies using relevant mouse models will be required to reveal roles of IGF1 and leptin signaling in radiation-related inflammatory bowel disease and colorectal cancer [52-54].

In summary, our results, when considered along with the fact that IGF1, adiponectin, and leptin are multifunctional hormones with roles in immune function, energy homeostasis, cellular proliferation, metabolic regulation, and carcinogenesis [55, 56], have important implications not only for molecular understanding of radiation-induced chronic intestinal functional alterations and colorectal carcinogenesis but also for development of preventive strategies against long-term sequela of radiation exposure in GI tract. Also, considering that very little has been explored so far to reveal underlying mechanisms involved in non-lethal radiation-induced hormonal and metabolic perturbations in relation to GI disorders, our study, we believe, will provide further impetus to undertake research in this area involving varying radiation types and doses relevant to the clinics, genetic background, age, gender, and intervention strategies.

\section{Acknowledgements}

This study is supported in part by NASA Grant\# NNX13AD58G and NNX09AU95G. We acknowledge the Histopathology and Tissue Shared Resources at Georgetown University supported by Award Number P30CA051008 from the National Cancer Institute.

\section{Competing Interests}

The authors have declared that no competing interest exists.

\section{References}

1. Dupree-Ellis E, Watkins J, Ingle JN, et al. External radiation exposure and mortality in a cohort of uranium processing workers. Am J Epidemiol. 2000; 152:91-95.

2. Preston DL, Shimizu Y, Pierce DA, et al. Studies of mortality of atomic bomb survivors. Report 13: Solid cancer and noncancer disease mortality: 1950-1997. Radiat Res. 2003; 160:381-407.

3. Ritz B. Radiation exposure and cancer mortality in uranium processing workers. Epidemiology. 1999; 10:531-538.

4. Sont WN, Zielinski JM, Ashmore JP, et al. First analysis of cancer incidence and occupational radiation exposure based on the National Dose Registry of Canada. Am J Epidemiol. 2001; 153:309-318.

5. Gilbert ES, Omohundro E, Buchanan JA, et al. Mortality of workers at the Hanford site: 1945-1986. Health Phys. 1993; 64:577-590.

6. John BJ, Irukulla S, Abulafi AM, et al. Systematic review: adipose tissue, obesity and gastrointestinal diseases. Aliment Pharmacol Ther. 2006; 23:1511-1523.

7. Giovannucci E. Metabolic syndrome, hyperinsulinemia, and colon cancer: a review. Am J Clin Nutr. 2007; 86:s836-s842.

8. Fenton JI, Birmingham JM. Adipokine regulation of colon cancer: adiponectin attenuates interleukin-6-induced colon carcinoma cell proliferation via STAT-3. Mol Carcinog. 2010; 49:700-709.

9. Soubry A, Il'yasova D, Sedjo R, et al. Increase in circulating levels of IGF-1 and IGF-1/IGFBP-3 molar ratio over a decade is associated with colorectal adenomatous polyps. Int J Cancer. 2012; 131:512-517.

10. Mutoh M, Teraoka N, Takasu S, et al. Loss of adiponectin promotes intestinal carcinogenesis in Min and wild-type mice. Gastroenterology. 2011; 140:2000-8, 2008.e1.

11. Endo H, Hosono K, Uchiyama T, et al. Leptin acts as a growth factor for colorectal tumours at stages subsequent to tumour initiation in murine colon carcinogenesis. Gut. 2011; 60:1363-1371.

12. Drew JE, Farquharson AJ, Padidar S, et al. Insulin, leptin, and adiponectin receptors in colon: regulation relative to differing body adiposity independent of diet and in response to dimethylhydrazine. Am J Physiol Gastrointest Liver Physiol. 2007; 293:G682-G691.

13. Otake S, Takeda H, Fujishima S, et al. Decreased levels of plasma adiponectin associated with increased risk of colorectal cancer. World J Gastroenterol. 2010; 16:1252-1257.

14. Sridhar SS, Goodwin PJ. Insulin-insulin-like growth factor axis and colon cancer. J Clin Oncol. 2009; 27:165-167.

15. Schedlich LJ, Young TF, Firth SM, et al. Insulin-like growth factor-binding protein (IGFBP)-3 and IGFBP-5 share a common nuclear transport pathway in T47D human breast carcinoma cells. J Biol Chem. 1998; 273:18347-18352.

16. Giovannucci E. Insulin, insulin-like growth factors and colon cancer: a review of the evidence. J Nutr. 2001; 131:3109S-3120S.

17. Paz-Filho G, Mastronardi C, Franco CB, et al. Leptin: molecular mechanisms, systemic pro-inflammatory effects, and clinical implications. Arq Bras Endocrinol Metabol. 2012; 56:597-607.

18. Siegmund B, Lehr HA, Fantuzzi G. Leptin: a pivotal mediator of intestinal inflammation in mice. Gastroenterology. 2002; 122:2011-2025.

19. Yarandi SS, Hebbar G, Sauer CG, et al. Diverse roles of leptin in the gastrointestinal tract: modulation of motility, absorption, growth, and inflammation. Nutrition. 2011; 27:269-275.

20. Uddin S, Hussain AR, Khan OS, et al. Role of dysregulated expression of leptin and leptin receptors in colorectal carcinogenesis. Tumour Biol. 2013;

21. Donato JJ, Frazao R, Elias CF. The PI3K signaling pathway mediates the biological effects of leptin. Arq Bras Endocrinol Metabol. 2010; 54:591-602.

22. Vansaun MN. Molecular pathways: adiponectin and leptin signaling in cancer. Clin Cancer Res. 2013; 19:1926-1932.

23. Yoneda K, Tomimoto A, Endo H, et al. Expression of adiponectin receptors, AdipoR1 and AdipoR2, in normal colon epithelium and colon cancer tissue. Oncol Rep. 2008; 20:479-483.

24. Saxena A, Chumanevich A, Fletcher E, et al. Adiponectin deficiency: role in chronic inflammation induced colon cancer. Biochim Biophys Acta. 2012; 1822:527-536. 
25. Fenton JI, Birmingham JM, Hursting SD, et al. Adiponectin blocks multiple signaling cascades associated with leptin-induced cell proliferation in Apc Min/+ colon epithelial cells. Int J Cancer. 2008; 122:2437-2445.

26. Edwards-Bennett SM, Correa CR, Harris EE. Optimization of Adjuvant Radiation in Breast Conservation Therapy: Can We Minimize without Compromise? Int J Breast Cancer. 2011; 2011:321304.

27. Tavernier A, Cavin JB, Le Gall M, et al. Intestinal deletion of leptin signaling alters activity of nutrient transporters and delayed the onset of obesity in mice. FASEB J. 2014;

28. Datta K, Suman S, Kallakury BV, et al. Exposure to heavy ion radiation induces persistent oxidative stress in mouse intestine. PLoS One. 2012; 7:e42224.

29. Shillingford JM, Piontek KB, Germino GG, et al. Rapamycin ameliorates PKD resulting from conditional inactivation of Pkd1. J Am Soc Nephrol. 2010; 21:489-497.

30. Skaland I, Janssen EA, Gudlaugsson E, et al. Phosphohistone H3 expression has much stronger prognostic value than classical prognosticators in invasive lymph node-negative breast cancer patients less than 55 years of age. Mod Pathol. 2007; 20:1307-1315.

31. Tsai JS, Wu CH, Chen SC, et al. Plasma adiponectin levels correlate positively with an increasing number of components of frailty in male elders. PLoS One. 2013; 8:e56250.

32. Roszkowska-Gancarz M, Jonas M, Owczarz M, et al. Age-related changes of leptin and leptin receptor variants in healthy elderly and long-lived adults. Geriatr Gerontol Int. 2014;

33. Barker N. Adult intestinal stem cells: critical drivers of epithelial homeostasis and regeneration. Nat Rev Mol Cell Biol. 2014; 15:19-33.

34. Barker N, van Es JH, Kuipers J, et al. Identification of stem cells in small intestine and colon by marker gene Lgr5. Nature. 2007; 449:1003-1007.

35. Perrini S, Laviola L, Carreira MC, et al. The GH/IGF1 axis and signaling pathways in the muscle and bone: mechanisms underlying age-related skeletal muscle wasting and osteoporosis. J Endocrinol. 2010; 205:201-210.

36. Gao Q, Horvath TL. Cross-talk between estrogen and leptin signaling in the hypothalamus. Am J Physiol Endocrinol Metab. 2008; 294:E817-E826.

37. Fruhbeck G. Intracellular signalling pathways activated by leptin. Biochem J. 2006; 393:7-20.

38. Marwarha G, Prasanthi JR, Schommer J, et al. Molecular interplay between leptin, insulin-like growth factor-1, and beta-amyloid in organotypic slices from rabbit hippocampus. Mol Neurodegener. 2011; 6:41

39. Sharma D, Wang J, Fu PP, et al. Adiponectin antagonizes the oncogenic actions of leptin in hepatocellular carcinogenesis. Hepatology. 2010; 52:1713-1722.

40. Motobayashi $Y$, Izawa-Ishizawa $Y$, Ishizawa $K$, et al. Adiponectin inhibits insulin-like growth factor-1-induced cell migration by the suppression of extracellular signal-regulated kinase $1 / 2$ activation, but not Akt in vascular smooth muscle cells. Hypertens Res. 2009; 32:188-193.

41. Azzam EI, Jay-Gerin JP, Pain D. Ionizing radiation-induced metabolic oxidative stress and prolonged cell injury. Cancer Lett. 2012; 327:48-60.

42. Lee do $\mathrm{Y}$, Bowen $\mathrm{BP}$, Nguyen $\mathrm{DH}$, et al. Low-dose ionizing radiation-induced blood plasma metabolic response in a diverse genetic mouse population. Radiat Res. 2012; 178:551-555.

43. Jo SK, Seol MA, Park HR, et al. Ionising radiation triggers fat accumulation in white adipose tissue. Int J Radiat Biol. 2011; 87:302-310.

44. Nakamura S, Tanaka IB, Tanaka S, et al. Adiposity in female B6C3F1 mice continuously irradiated with low-dose-rate gamma rays. Radiat Res. 2010; 173:333-341.

45. Oeffinger KC, Mertens AC, Sklar CA, et al. Obesity in adult survivors of childhood acute lymphoblastic leukemia: a report from the Childhood Cancer Survivor Study. J Clin Oncol. 2003; 21:1359-1365.

46. Fan Y, Bergmann A. Apoptosis-induced compensatory proliferation. The Cell is dead. Long live the Cell! Trends Cell Biol. 2008; 18:467-473.

47. Alavi K, Schwartz MZ, Prasad R, et al. Leptin: a new growth factor for the small intestine. J Pediatr Surg. 2002; 37:327-330.

48. Ohneda K, Ulshen $\mathrm{MH}$, Fuller $\mathrm{CR}$, et al. Enhanced growth of small bowel in transgenic mice expressing human insulin-like growth factor I. Gastroenterology. 1997; 112:444-454.

49. Dube PE, Forse CL, Bahrami J, et al. The essential role of insulin-like growth factor-1 in the intestinal tropic effects of glucagon-like peptide- 2 in mice. Gastroenterology. 2006; 131:589-605.

50. Keku TO, Vidal A, Oliver S, et al. Genetic variants in IGF-I, IGF-II, IGFBP-3, and adiponectin genes and colon cancer risk in African Americans and Whites. Cancer Causes Control. 2012; 23:1127-1138.

51. Palmqvist R, Hallmans G, Rinaldi S, et al. Plasma insulin-like growth factor 1 , insulin-like growth factor binding protein 3 , and risk of colorectal cancer: a prospective study in northern Sweden. Gut. 2002; 50:642-646.

52. Moser AR, Luongo C, Gould KA, et al. ApcMin: a mouse model for intestinal and mammary tumorigenesis. Eur J Cancer. 1995; 31A:1061-1064.

53. Scheinin T, Butler DM, Salway $\mathrm{F}$, et al. Validation of the interleukin-10 knockout mouse model of colitis: antitumour necrosis factor-antibodies suppress the progression of colitis. Clin Exp Immunol. 2003; 133:38-43.

54. Datta K, Suman S, Kallakury BV, et al. Heavy ion radiation exposure triggered higher intestinal tumor frequency and greater beta-catenin activation than gamma radiation in APC(Min/+) mice. PLoS One. 2013; 8:e59295.

55. O'Connor JC, McCusker RH, Strle K, et al. Regulation of IGF-I function by proinflammatory cytokines: at the interface of immunology and endocrinology. Cell Immunol. 2008; 252:91-110.
56. Giovannucci E, Michaud D. The role of obesity and related metabolic disturbances in cancers of the colon, prostate, and pancreas. Gastroenterology. 2007; 132:2208-2225. 